\title{
The effects of diet composition and vitamin B supplementation on the urine isoprostane concentration in rats*
}

\author{
Z. Goluch-Koniuszy ${ }^{1}$ and J. Sadowska
}

\author{
West Pomeranian University of Technology in Szczecin, \\ Faculty of Food Sciences and Fisheries, Department of Human Nutrition Physiology \\ Papieża Pawta VI 3, 71-459 Szczecin, Poland
}

(Received 14 February 2012; revised version 30 August 2012; accepted 10 December 2012)

\begin{abstract}
The purpose of this research was to assess the amount of isoprostane 8 -iPF2 $\alpha$-III, a marker of oxidation stress, in excreted urine. The study was conducted on 40 male rats aged 5 months. Group 1 received the basic diet, groups 2-4, the modified diet in which whole cereal grains were partly substituted with wheat flour and sucrose. Groups 1-2 received water to drink, groups 3 and 4 were given aqueous solutions of B group vitamins at different doses. On the fifth week of the experiment, a 24-h urine collection was conducted. The concentrations of creatinine and 8-iPGF2a-III were determined in urine samples. Modification of diet composition caused increased excretion of isoprostane, which may indicate enhancement of free-radical reactions in the examined animal. The provided supplementation decreased the value of this parameter expressed as a creatinine ratio, but not, however, to the values observed in the animals fed the basic diet. A stronger effect was observed with excess supplementation.
\end{abstract}

KEY WORDS: rats, vitamins B, isoprostanes, urine

\section{INTRODUCTION}

The diets of many consumers are currently characterized by a high contribution of processed and purified food products rich in monosaccharides, with low contents of B-group vitamins, among others. About $25 \%-75 \%$ of the Polish population

\footnotetext{
* Supported by the Ministry of Science and Higher Education, Project No. 1248/PO1/2010/39

${ }^{1}$ Corresponding author: e-mail: zuzanna.goluch-koniuszy@zut.edu.pl
} 
supplements their diets with pharmaceutical preparations (Anuszewska, 2001), often at their own discretion and without any clear medical indications. Ample studies have shown that the level of supplementation frequently exceeds recommended levels by a few to several score times.

The available literature shows that the B-group vitamins have been in the focus of interest mainly owing to their deficiencies and their consequences both in humans under various physiological conditions, as well as in different animal species. Their effects have been determined in respect to blood and tissue biochemical markers, metabolic processes, and tolerable upper intake levels. In turn, investigations evaluating effects of an increased intake of synthetic forms of vitamins (supplements) refer most often to the impact of single vitamins or their complexes (Earnest et al., 2003), with the results frequently pointing to adverse effects of such intakes.

A diet rich in monosaccharides implies hyperglycaemia, which through mechanisms like glucose autoxidation, activation of polyalcohols, non-enzymatic glycation, and neutrophil granulocyte (GO) stimulation, induces production of ROS in amounts exceeding the physiological efficiency of the antioxidative system, thus leading to oxidation stress. Among the effect of oxidation stress is uncontrolled oxidation and peroxidation of lipids, i.e., generation of lipid peroxides and changes in the structure of polyunsaturated fatty acids in the phospholipids of cell- and mitochondrial membranes. This changes their liquidity, integrity, and functions (Evans et al., 2002). Accumulation of products being the effect of oxidation processes enlarges VCAM-1 expression and stimulates the mutual influence of monocytes and endothelial cells promoting early atheromatous changes, which can be prevented by antioxidants (Schmidt et al., 1995).

B-group vitamins, commonly used as dietary supplements, may exhibit antioxidative or prooxidative actions, depending on the dose or time of supplementation (Anand, 2005). A study by Friedrich and Dolot (2010) demonstrated that modification of diet composition, where whole wheat and maize grains were substituted with wheat flour and sucrose and the resultant diet was supplemented with vitamins $\mathrm{B}_{1}, \mathrm{~B}_{2}, \mathrm{~B}_{6}$, and niacin, resulted in a decrease of total antioxidative status and an increase in the malonyldialdehyde concentration in the blood of rats. Such markers as TAS (total antioxidative status), TBARS (thiobarbituric acid reactive substances), and MDA (malonyldialdehyde) are not, however, specific for the rat and do not provide an unambiguous answer as to the antioxidative defense potential of the body. The extent of oxidative stress in that species of animals can be explicitly indicated by the plasma concentration of 8 -iPF $2 \alpha$-III or the level of its urinary excretion (Roberts and Morrow, 2000). Increased concentrations/excretion of isoprostanes was demonstrated in many model studies conducted on animals with induced oxidative stress 
(Nanji et al., 1994), and the assay of isoprostane concentrations in tissues and/ or body fluids provides a biochemical basis for potential therapeutic intervention (Halliwell, 2000).

Therefore, in the conducted study, a hypothesis was advanced that the supplementation of a modified diet with B-group vitamins may enhance oxidative stress in animals, which will be manifested by increased urinary excretion of 8-iPF2 $\alpha$-III. As the effect of supplementation may be dose-dependent, two types of supplementation, complementary and excessive, were used in the study.

\section{MATERIAL AND METHODS}

\section{Animals and management}

Upon the approval of the Local Ethics Commission (Approval No. 2/2010), the study was conducted on 40 male Wistar rats aged 5 months with an initial body weight of $429 \pm 51 \mathrm{~g}$. After one-week conditioning under vivarium conditions (temp. $21-22^{\circ} \mathrm{C}$, relative air humidity $55 \%-60 \%$, light/dark cycle $12 / 12 \mathrm{~h}$ ), the animals were divided into four equal groups $(\mathrm{n}=10)$ that were fed ad libitum with pelleted diets produced from the same components, except for the differentiating ones, by the Feed Mixtures and Concentrates Production Plant in Kcynia (Poland). Group 1 received the basic diet (Labofed B) containing whole wheat and maize grain. Groups 2-4 were fed a modified diet in which $83.5 \%$ of the wheat in the basic diet was substituted with wheat flour type 500 , and $50 \%$ of the maize, with sucrose (Table 1). The contribution of the other components in the diets was identical. The substitution of dietary components to compose the modified diet was intended to mirror, to some extent, contemporarily observed nutritional mistakes, i.e., the increasing contribution of sucrose in the calorific value of a food ration and increased consumption of refined carbohydrates. The prepared diets were isocaloric and isoprotein (Table 1).

Groups 1-2 received water to drink, whereas the animals from group 3, during intensified activity, were given $25 \mathrm{ml}$ of an aqueous solution of vitamins in the following doses: $\mathrm{B}_{1}$ (thiamin hydrochloride) $0.94 \mathrm{mg}, \mathrm{B}_{2}$ (riboflavin) $0.48 \mathrm{mg}$, $\mathrm{B}_{6}$ (pyridoxine hydrochloride) $0.50 \mathrm{mg}$, niacin (nicotinamide) $1.9 \mathrm{mg}$, whereas in group 4 these doses equaled: $\mathrm{B}_{1} 3.1 \mathrm{mg}, \mathrm{B}_{2} 2.3 \mathrm{mg}, \mathrm{B}_{6} 2.4 \mathrm{mg}$, and niacin $6.65 \mathrm{mg}$. The vitamins were administered in quantities computed individually for the supplementation mode, i.e., in group 3, to supplement differences in the contents of those vitamins between diets resulting from the exchange of components (complementary supplementation), and in group 4, to make the administered dose exceed by 2 -4-fold (depending on the type of vitamin) the differences 
Table 1. Ingredients and nutrient composition in $100 \mathrm{~g}$ diets

\begin{tabular}{|c|c|c|}
\hline \multirow{2}{*}{ Indices } & Basic & Modified \\
\hline & \multicolumn{2}{|c|}{ diet } \\
\hline \multicolumn{3}{|l|}{ Ingredients, $g$} \\
\hline wheat grain, & 36.4 & 6 \\
\hline maize grain & 20 & 10 \\
\hline wheat bran & 20 & 20 \\
\hline dry whey & 3.0 & 3.0 \\
\hline $\mathrm{NaCl}$ & 0.27 & 0.25 \\
\hline soyabean meal $(48 \%)^{1}$ & 17 & 17 \\
\hline fodder chalk ${ }^{2}$ & 2 & 2 \\
\hline phosphate $1-\mathrm{CA}^{3}$ & 0.27 & 0.25 \\
\hline mineral and vitamin $\operatorname{mix}^{4}$ & 0.8 & 1 \\
\hline wheat flour type 500 & - & 30.4 \\
\hline saccharose & - & 10 \\
\hline \multicolumn{3}{|l|}{ Nutrient composition } \\
\hline total protein, g & 19.7 & 18.6 \\
\hline crude fat, g & 2.0 & 3.3 \\
\hline carbohydrates, g & 62.3 & 63.4 \\
\hline crude fibre, $g$ & 2.91 & 2.73 \\
\hline dry matter, g & 90.6 & 91.4 \\
\hline total ash, g & 6.61 & 6.17 \\
\hline thiamine, mg & 2.5 & 0.62 \\
\hline riboflavin, mg & 2.1 & 1.14 \\
\hline pyridoxine, mg & 2.35 & 1.35 \\
\hline niacin, mg & 8.6 & 4.8 \\
\hline \multicolumn{3}{|l|}{ gross energy } \\
\hline $\mathrm{kcal} \cdot \mathrm{g}^{-1}$ & 3.89 & 3.99 \\
\hline $\mathrm{kJ} \cdot \mathrm{g}^{-1}$ & 16.3 & 16.7 \\
\hline \multicolumn{3}{|l|}{ metabolizable energy } \\
\hline $\mathrm{kcal} \cdot \mathrm{g}^{-1}$ & 3.45 & 3.57 \\
\hline $\mathrm{kJ} \cdot \mathrm{g}^{-1}$ & 14.4 & 14.9 \\
\hline
\end{tabular}

${ }^{1}$ soyabean meal $48 \%$ - extracted, content $48 \%$ crude protein and $7 \%$ fibre; ${ }^{2}$ fodder chalk - content per kg: g: Ca 350, Mg 3,20; mg: Na 10.00, P 15.00; ${ }^{3}$ phosphate 1-CA - additive contains min. 22\% P and 15\% Ca; ${ }^{4}$ vitamin and mineral Premix LRM contents per kg: IU: A 1500000 , vit. $\mathrm{D}_{3}$ 100000 ; mg: vit. E 8000 ; vit. K 300 , vit. $\mathrm{B}_{1} 1200$, vit. $\mathrm{B}_{2} 1200$, vit. $\mathrm{B}_{6} 1000$, vit. $\mathrm{B}_{12} 8$, Se 100 , Fe 16000, Mn 4500, Zn 6000, Cu 1300, J 100, Co 200

between diets (excessive supplementation). Once the solution of vitamins was administered, the animals were provided with pure, settled tap water. After a oneweek conditioning period, the experiment lasted 6 weeks, during which feed intake in all groups as well as the vitamin intake in the supplemented groups was monitored systematically. The body weight of the animals was measured once a week. On the fifth week of the experiment, the experimental animals were placed in semimetabolic cages. After $48 \mathrm{~h}$ in the cages, a $24 \mathrm{~h}$-urine collection was conducted.

The animals were fasted $12 \mathrm{~h}$ before the end of the experiment. Next, they were anaesthetized with Ketanest and blood samples were collected from their hearts. 
Analysis

The prepared diets were subjected to chemical analysis (AOAC, 2003) to determine the contents of total nitrogen (by Kjeldahl's method) expressed in terms of protein content, crude fat (by Soxhlet's method), dry matter (by a gravimetric method), and ash (by a gravimetric method). The crude fibre content was also determined (in an ANKOM 220 apparatus). The carbohydrate content was calculated from the difference between dry matter and the sum of other solid dietary components. The gross and metabolizable energy contents were calculated using commonly applied energy equivalents.

In urine, creatinine concentrations were determined by a kinetic method using biotest kits from BioSystems. Urinary 8-iPGF2 $\alpha$-III was estimated with an enzyme-linked immunosorbent assay (EIA) kit from Cayman according to the manufacturer's instructions. The level of 8-isoprostane was standardized by expressing it as a creatinine ratio.

After centrifuging the coagulate, the resultant blood serum was used for determination of the glucose concentration by an enzymatic colorimetric technique. The amount of fat in the animals' livers was determined by Soxhlet's method on a Soxtec HT6 apparatus from Foss Tecator (AOAC, 2003).

The contents of vitamins $B_{1}, B_{2}, B_{6}$ and niacin in the diets were determined by extraction with ammonium in dimethylsulphoxide solution and acetic acid and HPLC with a DAD (using an Agilent 1200SL equipped with a diode array detector).

\section{Statistical analysis}

Statistical significance was assessed by one-way analysis of variance (ANOVA) using the Statistica 9.0 software package. When the ANOVA indicated a significant difference among the means, the differences were further evaluated using Duncan's multiple range tests.

\section{RESULTS AND DISCUSSION}

Analysis of the results demonstrated that both the modification of diet composition as well as diet supplementation with B-group vitamins had no significant effect on feed intake (Table 2). Likewise, a lack of the effect of diet supplementation with B-group vitamins on feed intake was demonstrated by Debski et al. (2007). Despite the known effect of a sucrose-containing diet on increasing body weight gains of animals (Chepulis, 2007), the reported study did 


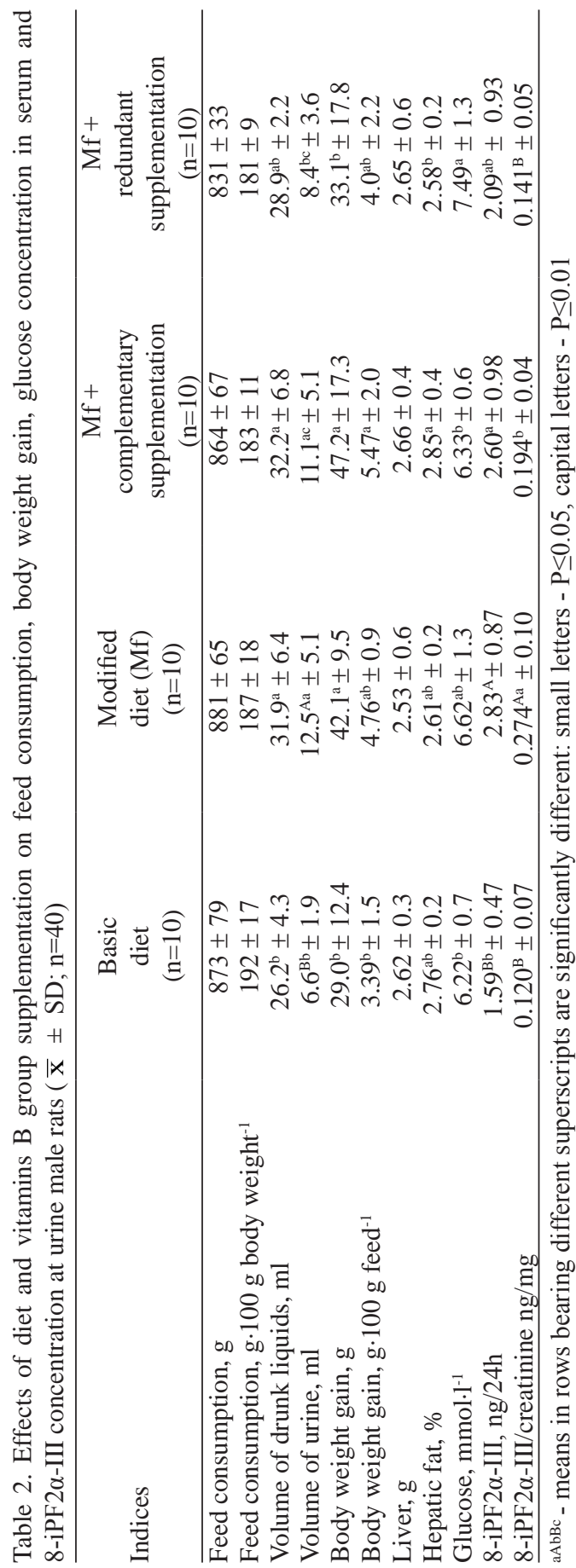


not demonstrate any statistically significant differences in body weight gains of the animals fed the modified diet containing sucrose compared with the animals fed the basic diet. A lack of such an effect was also reported by Toida et al. (1996). Both glucose and fructose provide carbon atoms and NADPH (the reduced form of nicotinamide adenine dinucleotide phosphate) for fatty acid synthesis. To assure the proper course of these metabolic pathways, however, appropriate quantities of B-group vitamins, which are co-factors in these metabolic processes, are indispensable. Hence, theoretically, when there is a deficiency of those vitamins, the synthesis of fats from carbohydrates may be inhibited. It should be noticed, however, that although the content of B-group vitamins in the modified diet corresponded to the demands of the animals (NRC, 1995), their lower supply than in the basic diet could also have been of significance.

The greatest body weight gains, both in absolute terms and per $100 \mathrm{~g}$ of feed intake, were found in the animals fed the modified diet supplemented with B-group vitamins in the complementary mode. The animals from this group were also characterized by a higher fat content in the liver. This may be due to several reasons. An inducing effect on hepatic fat synthesis is ascribed to the type of consumed carbohydrates (De Schrijver and Privett, 1983), because studies have shown that the highest biosynthesis of fat can be observed while feeding rats feed containing sucrose in comparison with feeds with a mix of carbohydrates or starch (De Castro et al., 2008).

The higher content of fat in the livers of rats fed the modified diet supplemented with B-group vitamins in the complementary mode may be explained by the availability of glucose in plasma. Fatty acids may be synthesized from plasma glucose and further be rapidly incorporated into acylglycerols of the microsomal fraction of hepatocytes and subsequently accumulated in intracellular droplets. The inclusion of thiamine among the supplemented vitamins might have channeled glucose metabolism into the pentose pathway, thus delivering pentoses for synthesis of NADPH. This process might have been enhanced by the presence of vitamin $\mathrm{B}_{6}$ among the supplementary vitamins that, by increasing magnesium absorption, stimulates the pentose pathway (Harripersad and Burger, 1997). The question why increased deposition of fat in the liver was not observed in the group of animals supplemented in the excessive mode still remains open. An expected outcome of the significantly higher plasma level of glucose, determined in this group of animals, should be an increase in the fat content of the liver, because studies (Morral et al., 2007) have demonstrated that in liver, hyperglycaemia causes the overexpression of a glucokinase gene, expression of genes of lipogenesis enzymes, and simultaneously suppresses the expression of genes taking part in fatty acid oxidation. On the other hand, however, the supplementation with niacin might have caused diminished esterification of fatty acids to triacylglycerols 
through an effect on mRNA expression and microsomal activity of diacylglycerol acyltransferase 2 (DGAT2) (Ganiji et al., 2004). The determined plasma glucose concentrations in the experimental animals were highest in those fed the modified diet supplemented with B-group vitamins in the excessive mode, and the differences compared with the groups fed the basic diet and the modified one supplemented in the complementary mode were statistically significant. The significant increase in the glucose concentration determined in the plasma of animals fed the modified diet supplemented with vitamins in the excessive mode might have been due to the presence of higher quantities of niacin than in the complementary supplementation. An excess of niacin in the diet causes an increase in the plasma glucose concentration, even despite concomitant supplementation with thiamine known for its normalizing effect on carbohydrate metabolism (Yenilmez et al., 2006).

Analysis of our results demonstrated a significant impact of diet modification on the quantity of ingested water, as well as an effect of diet modification and supplementation on the quantity of urine excreted by the animals (Table 2). The differences between the quantities of ingested fluids and the quantity of excreted urine were, however, comparable between the groups of animals. The analysis of daily excretion of 8 -iPF2 $\alpha$-III demonstrated that it was significantly higher in the group of animals fed the modified non-supplemented diet compared with those fed the basic diet. The complementary supplementation did not cause any changes in this respect, whereas the excessive supplementation significantly decreased the daily excretion of 8 -iPF $2 \alpha-$ III, however, these changes were statistically insignificant. Nevertheless, when expressing the concentration of 8 -iPF2 $\alpha$-III as a creatinine ratio, it was found that diet modification caused a statistically significant increase in the 8-iPF2 $\alpha$-III concentration, whereas diet supplementation decreased its concentration. It has already been demonstrated that disorders of the prooxidationantioxidation balance of the body, referred to as oxidative stress, are observed upon a higher concentration of glucose (Giugliano et al., 1996). In investigations by Friedrich et al. (2011), in a similarly constructed experiment, a decrease was noted in the activity of glutathione peroxidase (GSH-Px) in the blood of rats in response to both diet modification and its supplementation with B-group vitamins, which may indicate the enhancement of free-radical reactions. Peroxidation of lipids (Saito and Kubo, 2003), among others of polyunsaturated fatty acids (PUFA) built into membrane phospholipids, refers mainly to arachidonic acid, which as a free fatty acid is a precursor of eicosanoids, prostaglandins, tromboxanes and leukotrienes, synthesized in enzymatic pathways. When this acid is subjected to the non-enzymatic free radical mechanism of peroxidation, however, it is a precursor of the synthesis of isoprostanes, chemically stable isomers of prostaglandins that are released into the circulation through the action of A2 phospholipase and circulate therein in the free form or as esters in phospholipids of blood plasma 
(Roberts and Morrow, 2000). Isoprostane 8-iPF2 $\alpha$-III may also be formed in lipoprotein fractions (of LDL-cholesterol) in vivo and modified in vitro by the action of free radicals. Isoprostane 8 -iPF2 $\alpha$-III exerts strong vasospastic, mitogenic, as well as pro-adhesive and pro-aggregating effects on blood platelets (Gniwotta et al., 1997). In addition, it has already been shown that the increased excretion of isoprostanes with urine is significantly correlated with acholesterolaemia, hyperglycaemia and enhanced activity of AlAT (Davi et al., 1999) as well as diminished concentration of antioxidants (Nanji et al., 1994). In the reported experiment, the administration of the modified diet, in which whole grains of cereals were substituted with flour and sucrose, caused a significant increase in the concentration of 8 -iPF $2 \alpha$-III in urine. This effect may be due to insignificantly higher glycaemia generating reactive oxygen species, which leads to oxidative damage of lipid elements of cell membranes (Davi et al., 1999). The enhancement of freeradical reactions in the animals of this group might have resulted from the presence of sucrose in the modified diet. Busserolles et al. (2002) demonstrated in rats an increased intensity of lipid peroxidation in vivo, determined by the extent of TBARS excretion with urine, under the influence of sucrose in the diet. The unfavourable changes observed in this respect were explained by the presence of fructose (McDonald, 1995), which facilitates oxidative damage. The enhanced peroxidation of lipids, and thus the observed significant increase in the urinary concentration of 8 -iPF2 $\alpha$-III in the animals fed the modified diet, could be attributed to the nearly $75 \%$ lower content of vitamin $\mathrm{B}_{1}$ and over $42 \%-45 \%$ lower contents of $\mathrm{B}_{2}, \mathrm{~B}_{6}$, and niacin in that diet compared with the basic diet. Enhanced lipid peroxidation in the liver and peroxidation stress have been demonstrated in rats both upon thiamine (Sushko and Lukienko, 1981) and pyridoxine (Taysi, 2005) deficiency in diet. Despite the modified diet not being deficient in B-group vitamins, it was characterized by a lower content of these vitamins, which along with the increased content of sucrose could evoke the observed changes.

In turn, the lower urinary excretion of 8 -iPF $2 \alpha-$ III in the animals fed the modified diet supplemented with B-group vitamins, despite a significantly higher concentration of glucose in blood, may be explained by the effects of the administered vitamins. It has been demonstrated (Anand, 2005) that those vitamins cay display both antioxidative and pro-oxidative properties in respect to lipid peroxidation, depending on the length of their administration. Higashi-Okai et al. (2006) have demonstrated that thiamine, riboflavin, and niacin applied in the same concentration for a short period of time may express pro-oxidative effects, but upon long-term administration (likewise in the conducted 6-week experiment) may exhibit antioxidative action, and that pyridoxine shows strong antioxidative effects irrespective of the duration of its supplementation. 


\section{CONCLUSIONS}

In conclusion, modification of diet composition, in which whole cereal grains were partly substituted with wheat flour and sucrose, caused the increased excretion of 8 -iPF2 $\alpha$-III, which may indicate the enhancement of free-radical reactions in the examined animal. In turn, the applied supplementation with B-group vitamins significantly decreased the value of this parameter expressed as a creatinine ratio, however, not to the values observed in the animals administered the basic diet; a stronger effect in this respect was, however, observed with the excessive supplementation.

\section{REFERENCES}

Anand S.S., 2005. Protective effect of vitamin B6 in chromium-induced oxidative stress in liver. J. Appl. Toxicol. 25, 440-443

Anuszewska E.A., 2001. Pharmaceutical preparations containing vitamins and minerals recommended for children as a diet supplementation. Pediatr. Współcz. Gastroenterol. Hepatol. Żywienie Dziecka 3 (2), 145-151

AOAC, 2003. Association of Official Analytical Chemists, Official Methods of Analysis. 17 $7^{\text {th }}$ Edition. Gaithersburg, MD

Busserolles J., Rock E., Gueux E., Mazur A., Grolier P., Rayssiguier Y., 2002. Short-term consumption of a high-sucrose diet has a pro-oxidant effect in rats. Brit. J. Nutr. 87, 337-342

Chepulis L., 2007. The effect of honey compared to sucrose, mixed sugars and a sugar free diet on weight gain in young rats. J. Food Sci. 7, 224-229

Davi G., Ciabattoni G., Consoli A., Mezzetti A., Falco A., Santarone S., Pennese E., Vitacolonna E., Bucciarelli T., Costantini F., Capani F., Patrono C., 1999. In vivo formation of 8-isoprostaglandin F2 $\alpha$ and platelet activation in diabetes mellitus: effects of improved metabolic control and vitamin E supplementation. Circulation 99, 224-229

De Castro G.S.F., de Almeida L.P., Vannucchi H., Portari G.V., Jordão A.A., 2008. Effects of diets containing different types of carbohydrates on hepatic metabolism. Scand. J. Lab. Anim. Sci. $35,321-328$

De Schrijver R., Privett O.S., 1983. Hepatic fatty acids and acyl desaturases in rats: effects of dietary carbohydrate and essential fatty acids. J. Nutr. 113, 2217-2222

Debski B., Bertrandt J., Klos A., Gralak M., 2007. The influence of folic acid, vitamins $B_{2}$ and $B_{6}$ supplementation on feed intake, body and organs weight, and liver fatty acids composition of rats subjected to 3 months moderate protein deprivation. Vet. Med. A Physiol. Pathol. Clin. Med. 54, 57-61

Earnest C.P., Wood K.A., Church T.S., 2003. Complex multivitamin supplementation improves homocysteine and resistance to LDL-C oxidation. J. Amer. Coll. Nutr. 22, 400-407

Evans J., Goldfine I., Maddux B., Grodsky G.M., 2002. Oxidative stress and stress-activated signaling pathways: a unifying hypothesis of type 2 diabetes. Endocr. Rev. 23, 599-622

Friedrich M., DolotA., 2010. Assessment of effects of diet composition and vitamin B supplementation of free radical-related processes in the body. Activity of antioxidant enzymes and the total antioxidant status of rat blood. Pol. J. Food Nutr. Sci. 60, 281-287 
Friedrich M., Goluch-Koniuszy Z., Dolot A., Pilarczyk B., 2011. Appreciation of selenium concentration in blood and tissues of male rat as a result of diet ingredients changes and its supplementation with chosen group B vitamins. Rocz. Państ. Zakl. Hig. 162, 41-46

Ganji S.H., Tavintharan S., Zhu D., Xing Y., Kamanna V.S., Kashyap M.L., 2004. Niacin noncompetitively inhibits DGAT2 but not DGAT1 activity in HepG2 cells. J. Lipid Res. 45, 1835-1845

Giugliano D., Ceriello A., Paolisso G., 1996. Oxidative stress and diabetic vascular complications (Review). Diabetes Care 3, 257-267

Gniwotta C., Morrow J.D., Roberts L.J., Kuhn H., 1997. Prostaglandin F2-like compounds, F2isoprostanes, are present in increased amounts in human atherosclerotic lesions. Arterioscler. Thromb. Vasc. Biol. 17, 3236-3241

Halliwell B., 2000. Lipid peroxidation, antioxidants and cardiovascular disease: how should we move forward? Cardiovasc. Res. 47, 410-418

Harripersad R., Burger F.J., 1997. The effect of a subnormal dose of vitamin $B_{6}$ on plasma lipid in the rats. Int. J. Vitam. Nutr. Res. 2, 95-101

Higashi-Okai K., Nagino H., Yamada K., Okai Y., 2006. Antioxidant and prooxidant activities of B group vitamins in lipid peroxidation. J. UOEH 1, 359-368

McDonald R.B., 1995. Influence of dietary sucrose on biological aging. Amer. J. Clin. Nutr. 62, 284S-293S

Morral N., Edenberg H.J., Witting S.R., Altomonte J., Chu T., Brown M., 2007. Effects of glucose metabolism on the regulation of genes of fatty acid synthesis and triglyceride secretion in the liver. J. Lipid Res. 48, 1499-1510

Nanji A.A., Khwaja S., Tahan S.R., Sadrzadeh S.M., 1994. Plasma levels of a novel noncyclooxygenase-derived prostanoid (8-isoprostane) correlate with severity of liver injury in experimental alcoholic liver disease. J. Pharmacol. Exp. Ther. 269, 1280-1285

NRC, 1995. Nutrient Requirements of Laboratory Animals. $4^{\text {th }}$ revised Edition. National Academy Press. Washington, DC

Roberts L.J., Morrow J.D., 2000. Measurement of F2-isoprostanes as an index of oxidative stress in vivo. Free Radical Biol. Med. 28, 505-513

Saito M., Kubo K., 2003. Relationship between tissue lipid peroxidation and peroxidizability index after $\alpha$-linolenic, eicosapentaenoic, or docosahexaenoic acid intake in rats. Brit. J. Nutr. 89, $19-28$

Schmidt A.M., Hori O., Chen J.X., Li J.F., Crandall J., Zhang J., Cao R., Yan S.D., Brett J., Stern D., 1995. Advanced glycation endproducts interacting with their endothelial receptor induce expression of vascular cell adhesion molecule-1 (VCAM-1) in cultured human endothelial cells and in mice. A potential mechanism for the accelerated vasculopathy of diabetes. J. Clin. Invest. 96, 1395-1403

Sushko L.I., Lukienko P.I., 1981. Effect of vitamin B1 deficiency on xenobiotic hydroxylation and lipid peroxidation in rat liver microsomes. Farmakol. Toksikol. 2, 102-104

Taysi S., 2005. Oxidant/antioxidant status in liver tissue of vitamin B6 deficient rats. Clin. Nutr. 24, 385-389

Toida S., Takahashi M., Shimizu H., Sato N., Shimomura Y., Kobayashi I., 1996. Effect of high sucrose feeding on fat accumulation in the male Wistar rat. Obes. Res. 4, 561-568

Yenilmez A., Ozçifçi M., Aydin Y., Turgut M., Uzuner K., Erkul A., 2006. Protective effect of highdose thiamine (B1) on rat detrusor contractility in streptozotocin-induced diabetes mellitus. Acta Diabetol. 43, 103-108 